\title{
Malt Extract as a Healthy Substitute for Refined Sugar
}

\author{
Daniela F Seixas Chaves ${ }^{1 *}$, Daniela Valim Hatano ${ }^{2}$ and Dulcineia Rossini Milan ${ }^{2}$ \\ ${ }^{1}$ Department of Food Science and Experimental Nutrition, University of São Paulo, Brazil \\ ${ }^{2}$ Department of Research and Development, Liotécnica Tecnologia em Alimentos, Brazil
}

*Corresponding author: Daniela F Seixas Chaves, Department of Food Science and Experimental Nutrition, School of Pharmaceutical Sciences, University of São Paulo, São Paulo 03178-200, Brazil.

To Cite This Article: Daniela F Seixas Chaves. Malt extract as a Healthy Substitute for Refined Sugar. Am J Biomed Sci \& Res. 2019 - 4(1). AJBSR. MS.ID.000758. DOI: 10.34297/AJBSR.2019.04.000758

Received: July 12, 2019 | Published: July 17, 2019

\begin{abstract}
An overconsumption of refined added sugars may cause inflammation, insulin resistance and an increase in visceral adiposity. It is important to highlight that refined sugar is devoid of micronutrients and phytochemicals and natural sweeteners such as malt extract and honey have a different effect in oxidative stress markers and overall health. Malt extract contains approximately $90 \%$ carbohydrate, most of it in the form of maltose, B vitamins (mainly niacin), amino acids and some minerals such as magnesium and potassium. It is also rich in phenolic compounds mainly ferulic acid and flavan-3-ols. It has a high antioxidant activity, both in vitro and in vivo, which makes malt extract a healthy substitute for refined sugars.
\end{abstract}

Keywords: Sugar; HFCS; Malt Extract; Antioxidant

\section{Refined sugar and obesity}

An excessive caloric intake and physical inactivity is one of the main causes of obesity, but current research has shown that not only calories, but the type of calories such as the overconsumption of added sugars and refined carbohydrates induce insulin resistance and obesity [1].

The most common added sugars consumed by Americans, high-fructose corn syrup (HFCS) and sucrose, are made up of both fructose and glucose with a ratio of 50:50 for sucrose and 42\% or $55 \%$ of fructose for "HFCS 42 " and "HFCS 55", respectively.

Many studies have emphasized the differences in the metabolism of these monosaccharides: although glucose and fructose have the same molecular formula, their structure and metabolism are different. In contrast to glucose, the metabolism of fructose is not regulated by insulin nor at the enzymatic level, by phosphofructokinase. Therefore, an excess of fructose can lead to the formation of Acetyl Co-A, and an increase in lipogenesis, which may trigger several other events such as hypertriglyceridemia, hepatic steatosis and insulin resistance [2-5].

Although the harm of an excess of fructose is still a matter of debate and depends upon the consumption and the cardiometabolic endpoints evaluated [6], it is important to consider the food matrix as well. Honey composition is similar to HFCS considering only the ratio of monosaccharides, but differently from refined sugars, it contains a wide range of minor constituents such as vitamins, minerals, amino acids and phytochemicals, mainly phenolic acids and flavonoids [7,8]. Several studies highlight the composition of honey and show that differently from refined sugar, natural alternatives are not only not harmful, but beneficial. Detailing these benefits is beyond the scope of this review, and we suggest the excellent reviews already published on this topic: [9-13].

\section{Malt extract nutritional composition}

Malt extract is a hydrolysate of germinated barley grains. It contains approximately $90 \%$ carbohydrate, most of it in the form of maltose, B vitamins (mainly niacin), amino acids and some minerals such as magnesium and potassium [14]. Also, malt extract is rich in phenolic compounds with a total polyphenol content of $0.6-2.9 \mathrm{mg}$ GAE/gdw, of which the dominant compound is ferulic acid (ranging from $7.8-56.1 \mu \mathrm{g} / \mathrm{gdw}$ ) [15]. It is also a source of flavan-3-ols such as (+)-catechins and (-)-epicatechin [16].

Studies with malt extract suggest it may be a healthy substitute for refined sugar due to its content in micronutrients, phytochemicals and high antioxidant activity (both in vitro and in vivo). The North American Society for Pediatric Gastroenterology has considered malt extract as a safe osmotic laxative for children, without side effects [17]. 


\section{Antioxidant activity}

Increased oxidative stress can induce a variety of chronic health problems such as inflammation, insulin resistance and neurodegenerative disorders. A study conducted by Boden et al. [18] showed that the initial event that triggers insulin resistance is an oxidative modification of the glucose transporter GLUT4 (carbonylation), caused by excessive feeding. Therefore, they suggest that the link between overnutrition and insulin resistance is an increase in oxidative stress, which inactivates GLUT4 requiring a compensatory increase in insulin production.

Malt extract has a high antioxidant activity, both in vitro and in vivo, assessed by its ability to scavenge hydroxyl and superoxide radicals, thus protecting against oxidative damage. Its phenolic compounds are responsible for its effective antioxidant properties. In a mouse model of aging, malt extract showed an antioxidant effect decreasing the levels of liver and brain malondialdehyde and improving total antioxidant capacity. Furthermore, it prevented the decrease of the antioxidant enzymes SOD and GPx. These authors suggested malt extract should be considered as a new source of natural antioxidants for pharmaceutical or dietary needs [19].

Phillips et al. [20] rationalized that substituting the quantity of refined sugars consumed in an average Western diet (130g per person per day) for sugars rich in antioxidants could increase the consumption of phytochemicals and antioxidants, therefore contributing to a higher antioxidant capacity.

By using the FRAP assay they showed that date sugar, molasses (blackstrap and dark) and barley malt syrup had the highest antioxidant capacity. Malt syrup had a FRAP value of 1.5 mmol/100g (refined sugar had a FRAP of $0.01 \mathrm{mmol} / 100 \mathrm{~g}$ and honey, $0.165 \mathrm{mmol} / 100 \mathrm{~g}$ ). Based on these values they showed that substituting refined sugar for more natural sources would result in an average increase of $2.6 \mathrm{mmol} /$ day in antioxidants intake, which is equivalent to the consumption of a serving of antioxidant rich foods such as berries or nuts [20].

\section{Conclusion}

Although there is still a lack of clinical trials using malt syrup, its nutritional composition (micronutrients and phytochemicals) and its high antioxidant capacity measured both in vitro and in vivo suggest that it is a healthier substitute for refined sugar.

\section{References}

1. DiNicolantonio JJ, Mehta V, Onkaramurthy N, Keefe JH (2018) Fructoseinduced inflammation and increased cortisol: A new mechanism for how sugar induces visceral adiposity. Prog Cardiovasc Dis 61(1): 3-9.

2. Stanhope KL, Schwarz JM, Keim NL, Griffen SC, Bremer AA, et al. (2009) Consuming fructose-sweetened, not glucose-sweetened, beverages increase visceral adiposity and lipids and decreases insulin sensitivity in overweight/obese humans. J Clin Invest 119(5): 1322-1334.

3. Samuel VT (2011) Fructose induced lipogenesis: from sugar to fat to insulin resistance. Trends Endocrinol Metab 22(2): 60-65.
4. Teff KL, Elliott SS, Tschöp M, Kieffer TJ, Rader D, et al. (2004) Dietary fructose reduces circulating insulin and leptin, attenuates postprandial suppression of ghrelin, and increases triglycerides in women. J Clin Endocrinol Metab 89(6): 2963-2972.

5. Teff KL, Grudziak J, Townsend RR, Dunn TN, Grant RW, et al. (2009) Endocrine and metabolic effects of consuming fructose- and glucosesweetened beverages with meals in obese men and women: influence of insulin resistance on plasma triglyceride responses. J Clin Endocrinol Metab 94(5): 1562-1569.

6. Sievenpiper JL, de Souza RJ, Cozma AI, Chiavaroli L, Ha V, et al. (2014) Fructose vs. glucose and metabolism: do the metabolic differences matter? Curr Opin Lipidol 25(1): 8-19.

7. Escuredo O, Dobre I, Fernández-González M, Seijo MC (2014) Contribution of botanical origin and sugar composition of honeys on the crystallization phenomenon. Food Chem 149: 84-90.

8. Da Silva PM, Gauche C, Gonzaga LV, Ana Carolina OliveiraCosta, RoseaneFett (2016) Honey: Chemical composition, stability and authenticity. Food Chem 196: 309-323.

9. Ahmed S, Sulaiman SA, Baig AA, Muhammad Ibrahim, Sana Liaqat, et al. (2018) Honey as a Potential Natural Antioxidant Medicine: An Insight into Its Molecular Mechanisms of Action. Oxid Med Cell Longev p. 19.

10. Alvarez Suarez JM, Gasparrini M, Forbes-Hernández TY, Mazzoni L, Giampieri F (2014) The composition and biological activity of honey: A focus on Manuka honey. Foods 3(3): 420-432.

11. Erejuwa 00, Sulaiman SA, Wahab MS (2014) Effects of honey and its mechanisms of action on the development and progression of cancer. Molecules 19(2): 2497-2522.

12. Musa Özcan, M, Al Juhaimi F (2015) Honey as source of natural antioxidants. J Apic Res 54(3): 145-154.

13. Nguyen HTL, Panyoyai N, Kapasis S, Edwin Pang, Nitin Mantri (2019) Honey and its role in relieving multiple facts of atherosclerosis. Nutrients 11(1): 167.

14. Lougham DM (1997) Nutritional benefits of malt extract, Sandoz Nutrition Ltd.

15. Dvoráková M, Douanier M, Jurková M, Vladimír Kellner, Pavel Dostálek (2008) Comparison of Antioxidant Activity of Barley (Hordeum vulgare L.) and Malt Extracts with the Content of Free Phenolic Compounds Measured by High Performance Liquid Chromatography Coupled with CoulArray Detector. J Inst Brew 114(2): 150-159.

16. Goupy P, Hugues M, Boivin P, Amiot MJ (1999) Antioxidant composition and activity of barley (Hordeum vulgare) and malt extracts and of isolated phenolic compounds. J Sci Food Agric 79(12): 1625-1634.

17. Williams L, Philadephia W (2006) Evaluation and treatment of constipation in infants and children: Recommendations of the north American society for Pediatric Gastroenterology, hepatology and nutrition. J Pediatr Gastroenterol Nutr 43(3): e1-e13.

18. Boden G, Homko, Barrero CA, Stein TP, Chen X, et al. (2015) Excessive caloric intake acutely causes oxidative stress, GLUT4 carbonylation, and insulin resistance in healthy men. Sci Transl Med 7(304): 304re7.

19. Qingming Y, Xianhui P, Weibao K, YangHong, SuYidan, et al. (2010) Antioxidant activities of malt extract from barley (Hordeum vulgare L.) toward various oxidative stress in vitro and in vivo. Food Chem 118(1): 84-89.

20. Phillips KM, Carlsen MH, Blomhoff R (2009) Total antioxidant content of alternatives to refined sugar. J Am Diet Assoc 109(1): 64-71. 